\title{
Passer de la gêne à la prévention
}

\author{
Les adolescent-e-s lesbiennes, gays, bisexuel-le-s, transgenres (LGBT) subissent une \\ discrimination homophobe qui les rend vulnérables. Reconnaître les difficultés qu'ils \\ rencontrent, travailler sur les a priori des professionnel-le-s qui les entourent, \\ informer sur les ressources à disposition sont des moyens d'actions concrets qui \\ permettent de les soutenir, notamment en milieu scolaire.
}

\section{Elisabeth Thorens-Gauda, Dr Olivier Duperrex ${ }^{b}$}

a Attachée aux questions d'homophobie et de diversité pour les cantons de Vaud et de Genève

b Responsable de l'Unité de promotion de la santé et de prévention en milieu scolaire, Vaud, MD MSc, FMH en pédiatrie

\footnotetext{
* Les références se trouveront dans l'Internet sous www.bms.ch $\rightarrow$ Numéro actuel ou $\rightarrow$ Archives $\rightarrow 2012 \rightarrow 6$
}

Correspondance:

Unité PSPS

AVASAD

Route de Chavannes 37 Ouest

CH-1014 Lausanne

Tél. 041213163321

Fax 041213163330

elisabeth.thorens-gaud[at]vd.ch

\section{Introduction}

Dans le cadre de la semaine lausannoise contre le racisme placée sous le thème du sport, le Bureau lausannois pour l'intégration des immigrés BLI, organisait une conférence sur le thème de l'homophobie au mois de mars 2011 [1]*. Des participant-e-s issu-e-s des milieux de l'éducation et de la santé se sont réunis pour échanger autour de cette thématique.

Caroline Dayer, enseignante et chercheuse à la Faculté des sciences de l'éducation de l'Université de Genève, a donné des clés pour repérer les situations d'homophobie. Pierre-André Michaud, Professseur à l'Université de Lausanne et médecin chef de l'Université multidisciplinaire de santé des adolescents UMSA (CHUV), a expliqué les conséquences négatives que les discriminations homophobes pouvaient engendrer chez les adolescent-e-s, en s'appuyant sur des cas concrets rencontrés dans l'exercice de sa pratique. Elisabeth Thorens-Gaud, enseignante et attachée aux questions d'homophobie et de diversité pour les cantons de Vaud et de Genève a fourni des pistes aux participant-e-s pour les aider à promouvoir un climat ouvert à la diversité au sein de leurs institutions et clubs sportifs. Cet article reprend quelques éléments importants et offre quelques ressources utiles aux professionnel-le-s qui travaillent avec les adolescent-e-s.

\section{Les jeunes LGBT: une population vulnérable}

On estime que 5 à 10\% des adolescent-e-s et jeunes sont lesbiennes, gays, bisexuel-le-s, transgenres (LGBT). Ces jeunes LGBT ont un risque augmenté d'idées suicidaires, de tentatives de suicide, d'états dépressifs. De plus, leur consommation de tabac, d'alcool et de substances pourrait être plus élevée que celle des jeunes hétérosexuel-le-s et cisgenres. Les jeunes LGBT rapportent être victimes de violences et de harcèlement plus souvent que les jeunes hétérosexuel-le-s ou cisgenres notamment en milieu scolaire, ce qui a un impact sur leur vie d'adulte. Ces personnes présentent donc des «vulnérabilités spécifiques en terme de santé», sont souvent peu visibles et «sont nombreuses à avoir subi des violences psychologiques, verbales et/ou physiques» $[2-5]$.

\section{Méconnaissance des besoins des jeunes LGBT par le personnel soignant et enseignant}

Malgré l'évolution des mentalités, entre l'âge de 13 ans et 20 ans, les jeunes s'interrogeant sur leur genre et leur orientation sexuelle traversent des moments particulièrement difficiles à l'école, dans les milieux sportifs ainsi que dans les établissements médicaux qu'ils fréquentent. Le processus de coming out est encore largement méconnu. De même, il ne vient pas forcément à l'esprit du personnel encadrant qu'un jeune puisse avoir une orientation sexuelle autre qu'hétérosexuelle comme en témoigne Noémie:

"J'avais 18 ans, et j'ai fait une grosse dépression. J'ai été hospitalisée plusieurs fois. Durant un an, j'ai fait des aller-retour à l'hôpital. Même dans ce milieu hospitalier, je n'étais pas mieux. Je me retrouvais comme au collège, dans un monde complètement hétéro. On me considérait comme une hétéro par principe, ce qui m'a amenée à faire une tentative de suicide, alors que j'étais à l'hôpital.» [6]

\section{Incompréhension des familles et risque de rejet}

Facteur parfois aggravant, contrairement aux jeunes des autres minorités qui peuvent compter sur l'appui de leur famille quand ils sont discriminés, les jeunes LGBT sont souvent confrontés à un univers familial peu soutenant, voire menaçant. Le rejet de certains parents peut prendre des formes extrêmement graves: indifférence, négligence, mauvais traitements, expulsion du domicile familial. La famille ne constitue ainsi plus le facteur de protection attendu pour les jeunes LGBT. Témoignage de Mathieu:

«Ma mère l'a d'abord très mal pris, mais depuis elle fait son petit bonhomme de chemin. Mon père, quant à lui, a eu une réaction terrible; il m'a carrément rejeté. Un soir, il m'a saisi par les épaules, a fait mine de m'étrangler en me disant: Je te préviens, si tu es homo, je te tue et je me tue après! La violence de sa réaction m'a anéanti. 
A quoi bon se battre pour s'accepter soi-même, se faire accepter de ses amis, si ceux qui comptent le plus pour vous, votre famille, vous rejettent? Un seul geste, une seule parole avaient suffit à balayer tous mes efforts. J'étais totalement désespéré. J'ai pleuré toute la nuit. J'ai pensé au suicide, oui voilà.» [7]

Révélateurs, les deux exemples ci-dessus démontrent pourquoi il est nécessaire de promouvoir un climat ouvert à la diversité pour que les personnes LGBT puissent construire une image positive d'elles-mêmes. Les professionnel-le-s de l'éducation et de la santé ont donc le devoir de s'informer sur les besoins de ces jeunes. diverses instances en Suisse romande. Divers évènements de sensibilisation ont depuis été organisés dans plusieurs cantons romands [8-10].

Fermement déterminés à lutter contre l'homophobie en milieu scolaire et à promouvoir un climat d'établissement propice à l'épanouissement des élèves LGBT, le Conseiller d'Etat Charles Beer, Chef du Département de l'instruction publique, de la culture et du sport, DIP, Genève, ainsi que la Conseillère d'Etat Anne-Catherine Lyon, Cheffe du Département de la formation, de la jeunesse et de la culture, DFJC, Vaud ont engagé en 2010, une attachée aux questions d'homophobie et de diversité, puis ont défini, un plan

\section{«Ces jeunes LGBT ont un risque augmenté d'idées suicidaires, de tentatives de suicide, d'états dépressifs.»}

\section{Des actions pour prévenir I'homophobie dans les milieux éducatifs et de la santé} Une dynamique initiée par la Fédération genevoise des associations LGBT, qui avait organisé les Premières Assises contre l'homophobie en 2009, avec le soutien du canton de Genève, de la Ville de Genève et de l'Université de Genève, a permis de mobiliser

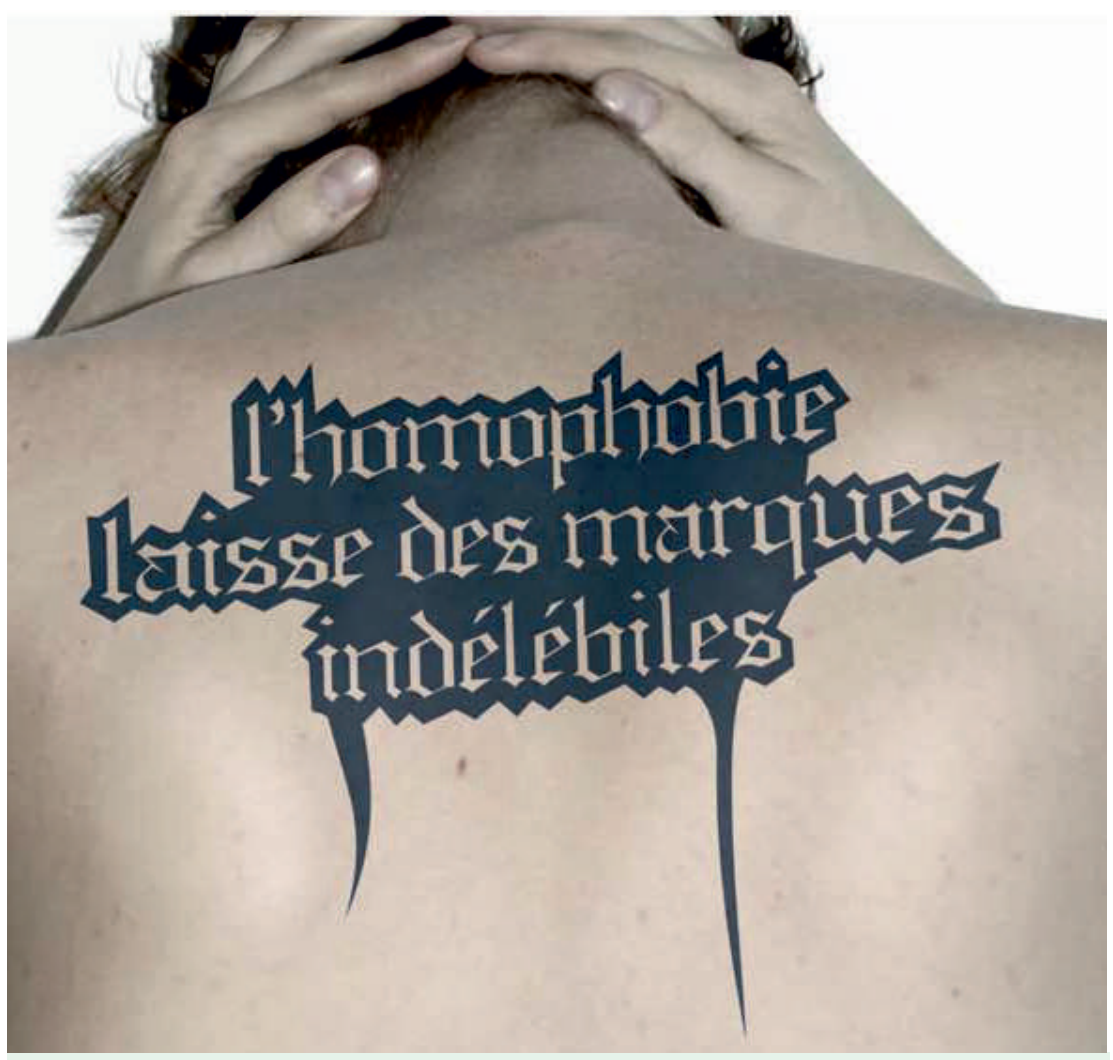

On estime que 5 à $10 \%$ des adolescent-e-s et jeunes sont lesbiennes, gays, bisexuel-le-s ou transgenres. (c) mosaic-info. Auteurs: Christophe Chênes et Cécilia Müller; $2^{e}$ prix du jury artistique. cadre pour mettre en place un dispositif d'interventions, des procédures et des offres de formation par le biais des services en charge de la promotion de la santé et de prévention en milieu scolaire (Vaud: Unité de Promotion de la santé et de prévention en milieu scolaire, Unité PSPS. Genève: Service santé jeunesse, SSJ).

\section{Une ressource pour les jeunes et les adultes qui les entourent}

Les cantons de Vaud et de Genève soutiennent également la plateforme d'information www.mosaicinfo.ch qui s'adresse aux jeunes, aux intervenant-e-s en milieu scolaire et aux parents. Ce site fournit des outils pour lutter contre les préjugés liés à l'orientation sexuelle et l'identité de genre. Il permettra notamment dans le futur la mise en commun de ressources entre les professionnel-le-s de la santé et de l'éducation. Depuis le début du mois d'octobre 2011, cette plateforme possède une extension pour les jeunes: www.mosaicjeunes.ch. Piloté par un jeune étudiant, ce portail répond aux besoins exprimés par les adolescent-e-s: briser l'isolement et offrir des ressources en ligne.

\section{Une série d'actions citoyennes pour sensibiliser la population}

Pour faire reculer l'homophobie, les cantons de Vaud et de Genève ont lancé un concours d'affiches au mois de novembre 2010. S'adressant aux Suisses romands âgés de 16 à 25 ans, ce concours a permis à 90 jeunes de s'exprimer sur cette discrimination. Les prix ont été décernés par un jury artistique, présidé par Pierre Keller, ancien Directeur de l'Ecole cantonale d'art de Lausanne ECAL, ainsi que par un jury pédagogique, composé de représentant-e-s vaudois-e-s et genevois-e-s de la formation et de la prévention en milieu scolaire. Le jury pédagogique a notamment désigné l'affiche actuellement utilisée pour la campagne d'affichage publique de prévention contre l'homo- 
phobie qui a débuté dans le canton de Vaud le 12 octobre 2011 et qui se poursuivra au début du mois de mars 2012 dans le canton de Genève.

Des moments de débats citoyens ponctuent cette campagne (soirée de remise des prix au théâtre de la Comédie à Genève, table ronde à l'Université de Lausanne et conférence à l'Université de Genève). Essentiels, ils permettent de toucher indirectement l'environnement social et familial de ces jeunes. La visibilité médiatique, qui accompagne généralement ces événements, contribue probablement elle aussi à faire évoluer les mentalités.
LGBT et le prestataire de l'éducation sexuelle en milieu scolaire PROFA, elle prendra en compte les recommandations de bonnes pratiques à adopter en milieu scolaire émises par les membres des groupes de travail qui ont œuvré à la mise sur pied des journées PREOS à Lausanne les 11 et 12 novembre 2011 [9]. Une sensibilisation est notamment prévue pour les cadres et des équipes de promotion de la santé et de prévention en milieu scolaire (médecins scolaires, infirmières scolaires, médiateurs et médiatrices scolaires, délégué-e-s PSPS), soit environ 600 personnes.

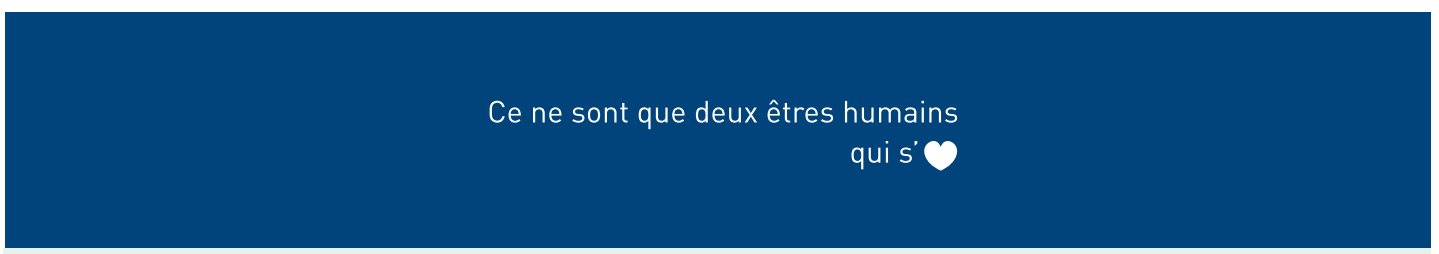

(c) mosaic-info. Auteur: Florian Montfort; $1^{\mathrm{er}}$ prix du jury pédagogique. Affiche retenue pour la campagne d'affichage publique.

L'exposition itinérante Stop homophobie conçue à partir des 19 créations lauréates du concours sera proposée aux établissements scolaires et de formation désirant mettre en place un projet d'établissement. Ces derniers pourront se faire accompagner dans leur démarche et bénéficier d'outils pédagoqiques conçus par des représentant-e-s des associations LGBT, de pédagogues et de spécialistes en promotion de la santé.

\section{Un cadre clair pour les intervenant-e-s en milieu scolaire}

Dans un message qu'il a adressé à toutes les directions scolaires du canton de Genève au mois d'octobre 2011, le magistrat Charles Beer a demandé qu'on encourage les actions et les procédures pour faire reculer l'homophobie dans les établissements. Il a aussi décidé que le Réseau d'alliés* devienne une priorité de l'action de son département. Le Service de santé de la jeunesse a déjà sensibilisé 73 professionnel-le-s à la diversité des identités des genres et des orientations sexuelles, soit $3 / 4$ de ses effectifs, sur une base de volontariat, lors de 3 journées d'atelier-formation en 20102011.

Comme l'a relevé la Conseillère d'Etat AnneCatherine Lyon, «il est important que dans chaque école, un adulte au moins, puisse entendre la demande, voire le SOS qu'un-e jeune lui adresserait.»** Pour répondre à ces besoins particuliers, l'Unité PSPS se voit confier le mandat de préparer la mise en œuvre d'actions en milieu scolaire pour prévenir l'homophobie, sensibiliser aux diversités de genre et d'orientation sexuelle et soutenir les élèves en questionnement ou LGBT. En collaboration avec les associations
Les cantons de Vaud et de Genève ont ainsi relevé le défi de mettre en place un dispositif de prévention de l'homophobie en milieu scolaire en Suisse. A cet égard, ils sont des pionniers. En principe, dans très peu d'années, les élèves de ces cantons devraient pouvoir grandir dans un environnement ouvert à la diversité.

\section{Et moi, que puis-je faire?}

Ne l'oublions pas, le respect de la dignité des personnes et des droits humains relève tant de notre responsabilité professionnelle que de notre responsabilité citoyenne.

Quelque soit son rôle, chacun peut au moins se questionner sur ses représentations. Plusieurs actions très concrètes sont proposées aux soignant-e-s par Bize et al. [4]. Nous vous proposons quelques sites internet en complément des références bibliographiques. Vous les trouverez dans l'Internet sous www. bms.ch $\rightarrow$ Numéro actuel.

\section{Remerciements}

Nous remercions pour leur soutien et leurs commentaires: Jean Schaer, responsable du réseau des délégué-e-s santé de l'Unité PSPS (VD); Serge Loutan, chef du Service de l'enseignement spécialisé et de l'appui à la formation au Département de la formation, de la jeunesse et de la culture (SESAF, DFJC, VD); Franceline Dupenloup, secrétaire adjointe au Département de l'instruction publique, de la culture et du sport (DIP, $\mathrm{GE})$; Dr Jean-Dominique Lormand, directeur du Service de santé de la jeunesse (SSJ, GE) et Prof. PierreAndré Michaud, médecin chef de l'Unité multidisciplinaire de santé des adolescents (UMSA, CHUV). 


\section{Références}

1 Bureau lausannois pour l'intégration des immigrés. Prévention Racisme - Semaine d'actions contre le racisme 2011. www.lausanne.ch/view. asp?DocId=34298 (updated 2011, cited 17.12.2011).

2 Institute of Medicine (US) Committee on Lesbian GBaTHIaRGaO. The Health of Lesbian, Gay, Bisexual, and Transgender People: Building a Foundation for Better Understanding. Washington (DC): 2011. www.ncbi.nlm.nih.gov/books/NBK64806/

3 Russell ST, Ryan C, Toomey RB et al. Lesbian, gay, bisexual, and transgender adolescent school victimization: implications for young adult health and adjustment. J.Sch Health. 2011;81:223-30. DOI: http://dx.doi.org/10.1111/j.1746-1561.2011.00583.x

4 Bize R, Volkmar E, Berrut $\mathrm{S}$ et al. Vers un accès à des soins de qualité pour les personnes lesbiennes, gays, bisexuelles et transgenres. Rev Med Suisse. 2011;7:1712-7. http://rms.medhyg.ch/ numero-307-page-1712.htm

5 Charriere E, Berrut S, Bize R et al. Diversité sexuelle et santé scolaire: des fragilités encore largement méconnues. Krankenpfl Soins Infirm. 2011;104:47-9. www.sante-plurielle.ch/fichier/divers/2011_09_soins_ infirmiers.pdf

6 TSR. Temps présent: Mon enfant est homo. www.tsr.ch/emissions/temps-present/1967912mon-enfant-est-homo.html (updated 24.6.2010, cited 17.12.2011).

7 Thorens-Gaud E. Adolescents homosexuels. Des préjugés à l'acceptation. Lausanne: Favre SA; 2009.

8 Fédération genevoise des associations LGBT. Premières assises contre l'homophobie à Genève - 4 et 5 septembre 2009. www.federationlgbt-geneve.ch/ (updated 2009).

9 PREOS: journées de réflexion et d'action sur la prévention du rejet basé sur l'orientation sexuelle et l'identité de genre - 11 et 12 novembre 2011 Lausanne. www.preos.ch (updated 2011).

10 Fédération genevoise des associations LGBT. Journée de Suivi des premières assises contre l'homophobie. www.federationlgbt-geneve.ch (updated 2011, cited 21.12.2011).

\section{Liens utiles}

- Unité de Promotion de la santé et de prévention en milieu scolaire Unité PSPS (VD) www.vd.ch/ unite-psps

- Unité Multidisciplinaire de Santé des Adolescents UMSA (VD) www.umsa.ch/

- Service Santé Jeunesse SSJ (GE) www.ge.ch/ssj

- Consultation Santé Jeunes HUG (GE) http:// sante-jeunes.hug-ge.ch

- Plateforme d'information pour les jeunes, les intervenant-e-s en milieu scolaire et les parents. Fournit des ressources et des informations en ligne sur la diversité sexuelle. www.mosaic-info.ch

- Fondation PROFA. Centre de références en matière de santé sexuelle et reproductive du canton de Vaud. www.profa.ch

- Site d'aide et d'information pour les adolescents. www.ciao.ch

- www.vogay.ch (VD)

- www.associationlilith.ch (VD)

- www.dialogai.org / Responsable du réseau d'alliés (GE)

- www.federationlgbt-geneve.ch (GE)

- La mission de la fondation Agnodice est de promouvoir en Suisse une société bienveillante et juste envers toute personne manifestant une identité de genre atypique.www.agnodice.ch

- Intervention sur la thématique de l'orientation sexuelle et de l'identité de genre. www.laboussole.ch 